\title{
STRATEGIE
}

\section{Kommunalisierung erfordert kommunales Lobbying}

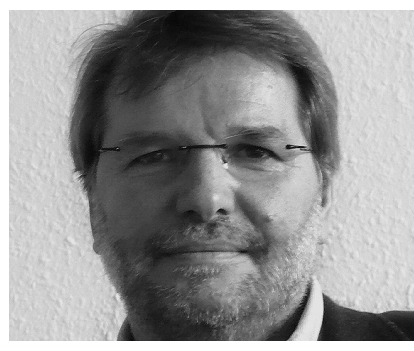

VON GÜNTER RIEGER Prof. Dr. Günter Rieger, Diplom Sozialpädagoge (BA) und Politikwissenschaftler (MA), ist Dekan der Fakultät Sozialwesen der Dualen Hochschule BadenWürttemberg Stuttgart sowie Studiengangsleiter "Soziale Dienste in der Justiz«. Sein Forschungsschwerpunkt ist die Sozialarbeitspolitik.

E-Mail rieger@dhbw-stuttgart.de
Soziale Arbeit braucht neben fachlich begründeten und wirtschaftlich gerechneten Konzepten auch ein professionelles Lobbying, um diese durchsetzen zu können. Dabei spielt die kommunale Ebene zunehmend eine größere Bedeutung. Eine erfolgreiche Lobbytätigkeit ruht dabei auf vier Säulen: Monitoring, Netzwerkarbeit, Politikberatung und Öffentlichkeitsarbeit.

Soziale Arbeit ist eine »Policy-Based Profession « (Popple/Leighninger 1998). Zu Recht spricht Thomas Olk von ihrer »Politikimmanenz«. Aufträge, Handlungsspielräume, Ressourcen oder Qualitätsstandards Sozialer Arbeit, ihrer Träger und Einrichtungen, sind immer auch das Ergebnis politischer Prozesse und Entscheidungen. Wo, wie und in welchem Umfang Soziale Arbeit hilft, ist selbstverständlich abhängig von fachlich methodischen Überlegungen und ökonomischen Kalkülen, aber stets ebenso Ergebnis politischer Willensbildung.

Eine besondere Rolle bei der Gestaltung Sozialer Arbeit spielt traditionell die Kommunalpolitik. Zwar wird in erster Linie im Bund und zunehmend auf europäischer Ebene über die Ausrichtung der Sozialpolitik, ihre gesetzlichen Rahmenbedingungen und ihre Finanzierung entschieden, aber die Kommunen sind im Gefüge des subsidiär organisierten bundesdeutschen Sozialstaates für die konkrete Leistungserbringung in sozialen Diensten und Einrichtungen verantwortlich. Dabei sind sie keine passiven Ausführungsorgane, welche sozialpolitische Vorgaben lediglich administrieren. Bei der notwendigen Allgemeinheit und Unbestimmtheit übergeordneter Gesetze und Programme fällt den Kommunen als Selbstverwaltungsorganen die Aufgabe zu, sozialstaatliche Vorgaben zu konkretisieren, anzupassen und zu ergänzen. Sowohl in ihren sozialgesetzlich verankerten Pflichtaufgaben wie auch im Rahmen freiwilliger Aufgaben haben die Kommunen dafür erhebliche Gestaltungsspielräume:

- Die Kommunen erfüllen in diesem Sinne eine »Optimierungsfunktion « (vgl. i. F. Bogumil/Holtkamp 2006: S. 218): Selbst dort, wo die Kommunen Pflichtleistungen erbringen, besteht ein erheblicher
Gestaltungsspielraum. Art und Umfang der Leistungen sind hier zwar durch die übergeordneten politischen Ebenen (Bund, Land) weitgehend festgelegt. Den Kommunen obliegt es aber, wie die zuständigen Ämter (insbesondere Jugendund Sozialamt) organisiert sind; ob die Leistungserbringung zentral oder dezentral erfolgt; wie weit hierarchische Steuerung beibehalten oder durch neue Steuerungsmodelle ergänzt wird; wie Qualität gesichert und kontrolliert wird; wie und wie viel Personal rekrutiert, fortgebildet und beteiligt wird; an welchem Leitbild die Arbeit ausgerichtet und welche fachliche Orientierung (Case Management, Sozialraumorientierung, integrierte Versorgung usw.) implementiert wird.

- Die Kommunen haben darüber hinaus eine »Integrationsfunktion «: Sozialpolitisches Handeln ist auf kommunaler Ebene auch dort gefragt, wo es gilt im Rahmen der Gewährleistungspflicht der Kommunen und orientiert am Subsidiaritätsprinzip die soziale Infrastruktur zwischen öffentlichen, freigemeinnützigen und gewerblichen Leistungserbringern zu gestalten. Setzt die Kommune auf Konkurrenz als marktwirtschaftliches Steuerungsmedium oder bevorzugt sie korporatistische Aushandlungsprozesse mit den Wohlfahrtsverbänden und Trägern der freien Jugendhilfe? Wie werden die unterschiedlichen Anbieter koordiniert und vernetzt? Welche Formen der Zusammenarbeit sind institutionalisiert? Wie wird die Leistungserbringung kontrolliert und der fachliche Austausch gewährleistet? Wie und in welcher Qualität wird Sozialplanung (Altenhilfe-, Jugendhilfeplanung usw.) durchgeführt? Wie ist der Zugang für neu entstehende Initiativen möglich und wie wird Selbsthilfe und Bürgerengagement einbezogen?

- Schließlich haben die Kommunen eine "Innovationsfunktion «: Im Rahmen freiwilliger Leistungen bestehen aufgrund der »Allzuständigkeit « der Kommunen vielfältige Möglichkeiten das Soziale zu gestalten (vgl. Dettling 2001). Auch wenn der Spielraum aufgrund gesetzlicher Regelungen wie schwacher Fi- 
nanzausstattung in den letzten Jahren enger geworden ist, zeigen vielfältige kommunale Initiativen und Modellprojekte, welche Möglichkeiten kommunale Sozialpolitik über die Erfüllung ihrer Pflichtaufgaben hinaus hat. Deutlich wird, dass eine kreative kommunale Sozialpolitik trotz externer Normierung und finanzieller Restriktionen einen Unterschied macht. Kommunale Sozialpolitik kann sich auf das gesetzlich Vorgeschriebene beschränken oder versuchen unter den gegebenen Bedingungen Sozialpolitik zu gestalten. Initiativen können in Rat und Verwaltung angestoßen (»top down «) werden, oder aus der Bürgerschaft (»bottom up «) kommen; kreative Mittelerschließung kann einen Finanzierungsmix aus Geldern unterschiedlicher staatlicher Ebenen (Europa, Bund, Länder), aus der Wirtschaft (Spenden, Social Sponsoring usw.) und der Zivilgesellschaft (Spenden, [Bürger-]Stiftungen usw.) ermöglichen; neue Steuerungsund Kooperationsformen können ausprobiert (private-public-partnership, Netzwerke, Markt usw.) werden.

Dabei nehmen die sozialpolitischen Gestaltungsnotwendigkeiten in den Kommunen aufgrund der den aktivierenden Sozialstaat kennzeichnenden Kommunalisierungsstrategie der Verlagerung sozialstaatlicher Aufgaben (vgl. Arbeitsförderung, Behindertenhilfe usw.) auf die Kommunen noch zu. Dieser Aufgabenzuwachs und die entsprechenden Gestaltungsnotwendigkeiten treffen aber in Zeiten der Wirtschaftskrise auf angespannte kommunale Haushalte mit der Konsequenz entsprechender Verteilungskämpfe. Beides erhöht die Notwendigkeit für die sozialen Verbände und Einrichtungen sich politisch einzumischen und politisch mit zu gestalten. Soziale Arbeit hatte und hat ein vitales Interesse an der Beeinflussung kommunalpolitischer Entscheidungen.

Allerdings haben sich Chancen und Formen möglicher Einflussnahme in der kommunalen Politikarena seit dem Ausgang des vorigen Jahrhunderts tiefgreifend verändert. Bis in die 80er Jahre des 20. Jahrhunderts war das kommunale Politikfeld im Sinne eines »lokalen Korporatismus « verfasst. Politische Strukturen und Prozesse waren von einer engen Durchdringung und Verflechtung von Wohlfahrtsverbänden und Räten wie Sozialverwaltungen gekennzeichnet. Sozialverbände und ihre Einrichtungen waren exklusiv und selbstverständlich in Formulierung und Ausführung von Politik eingebunden. Kommunale Sozialpolitik gestaltete sich vor dem Hintergrund wohlfahrtsverbandlich abgesteckter Claims, abgesichert durch personale Verflechtungen und eingebettet in spezifische Milieus. Verkrustung, Intransparenz und Exklusi- vität dieses Systems wurden seit den 1980er Jahren vor dem Hintergrund kommunaler Finanzprobleme, tiefgreifenden gesellschaftlichen Wandels und genereller Wohlfahrtsstaatskritik zunehmend kritisiert. Abwertend wurde vom » Wohlfahrtskartell « gesprochen.

Die zentrale Stellung der Wohlfahrtsverbände im subsidiären System Sozialer Sicherung blieb erhalten, doch die starren allerdings auch verlässlichen - korporativen Strukturen haben sich seit den 1980er Jahren aufgelöst. Gelockert wurde das korporatistische Arrangement von zwei Seiten. Einerseits sahen und sehen sich die Kommunen aufgrund von Finanzierungskrisen, wachsenden sozialen Ausgaben (Arbeitslo- sich dadurch nachhaltig. Gefordert ist die Implementierung marktwirtschaftlicher Mechanismen ebenso wie die Aktivierung, Einbeziehung und Koordinierung der Zivilgesellschaft. Doch kann schon aufgrund der Gewährleistungsfunktion der Kommune auf die klassisch hierarchische Steuerung über Rat und Verwaltung nicht verzichtet werden. Den entstehenden Steuerungsmix aus Markt, politischer Macht und zivilgesellschaftlicher Partizipation bezeichnet man als »local governance". Im neuen Spannungsfeld zwischen Markt, Politik und Zivilgesellschaft haben sich soziale Akteure heute zu orientieren, wenn sie erfolgreich Politik machen wollen. In diesem Umfeld braucht Soziale Arbeit fachlich

\section{"Im Sozialbereich haben die Kommunen vor allem drei Aufgaben: Optimierung, Integration, Innovation“}

sigkeit, demografischer Wandel usw.) und europäischer Wettbewerbspolitik in einem Klima neoliberaler Bürokratie- und Wohlfahrtsstaatskritik gezwungen, mehr marktwirtschaftliche Steuerungselemente einzuführen. Sozialverwaltungen wurden nach dem Modell der neuen Steuerung (»New Public Management «) reformiert. Dabei ging die Managerialisierung im Inneren der Verwaltung einher mit der Vermarktlichung und Privatisierung im Außenverhältnis. Dezentralisierung, Budgetierung und Ausschreibungen ordnen die Verhältnisse neu. Privatwirtschaftlich kalkulierende Sozialunternehmer treten als gleichberechtigte Konkurrenz freigemeinnütziger Träger auf.

Gleichzeitig wandelt sich die lokale Gesellschaft. Traditionelle Milieus lösen sich auf. Die vertikale, horizontale, soziale und ideelle Mobilität wächst. Partizipations- und Selbstbestimmungsansprüche nehmen zu, während traditionelle Engagementmotivationen zurückgehen. Neue Akteure (Bürgerinitiativen, Projektgruppen, Selbsthilfegruppen usw.) betreten selbstbewusst die Bühne und fordern Teilnahme und Teilhabe. Die Interessenpluralität nimmt zu, die kommunale Öffentlichkeit wird kritischer, sensibler und stimmungsabhängiger. Da sich die neuen Akteure aber weder über den Markt noch über die hierarchischen Strukturen der Verwaltung steuern lassen, gewinnen Vernetzungsstrategien und neue Partizipationsformen (Zukunftskonferenzen, Bürgerforen, Planungszellen usw.) an Bedeutung.

Die Möglichkeiten und Restriktionen kommunalpolitischer Steuerung verändern begründete und wirtschaftlich gerechnete Konzepte und ein professionelles Lobbying um sie durchzusetzen.

Die Pluralisierung der Interessen, Konkurrenz der Anbieter, gesteigerte Komplexität und Interdependenz gesellschaftlicher Handlungsfelder sowie eine zunehmend kritische Öffentlichkeit verändern die Chancen von Interessenvermittlung und Einflussnahme. Sie zwingen zur Professionalisierung.

\section{Was "Lobbying" meint}

Auf dem Gebiet sozialarbeiterischer Fachlichkeit und für das Sozialmanagement ist diese Entwicklung unübersehbar. Während die Bereiche des methodischen Arbeitens und das Wirtschaften der Sozialen Arbeit große Professionalisierungsfortschritte zu verzeichnen haben, ist ähnliches im Feld des "policy-making « kaum zu bemerken. Eine nachholende Professionalisierung ist hier ebenso wünschenswert wie unverzichtbar. Es gilt das kommunale Lobbying der Initiativen, Verbände und Einrichtungen Sozialer Arbeit fachlich zu entwickeln (vgl. Lallinger/Rieger 2007). Es gilt die politische Arbeit von Verbänden und Einrichtungen konzeptionell auszurichten, in Leitbildern, Organisationsstrukturen sowie Stellenbeschreibungen zu berücksichtigen und als Element der Qualität von Organisationen zu begreifen.

Lobbying bezeichnet alle direkten, informellen Versuche von Interessengruppen auf die Akteure des politischen Entscheidungsprozesses Einfluss zu nehmen, um Po- 
litikergebnisse in ihrem Sinne zu verändern. Professionelles Lobbying ist stets eine Tauschbeziehung. Es setzt den Aufbau und die Pflege vertrauensvoller Beziehungen voraus und beruht auf Glaubwürdigkeit, Zuverlässigkeit und Seriosität.

Im modernen Lobbying fließen Politikberatung, Netzwerk- und Öffentlichkeitsarbeit im Sinne eines »Public Affairs Management " zusammen. Ressourceneinsatz und Schaffung wie Ausbau von Ressourcen ergänzen sich im professionellen Lobbying. Denn Lobbytätigkeit muss auf bestimmte Ressourcen der eigenen Organisation zurückgreifen (Geld, Personal, Know-how usw.), generiert im Prozess des Lobbying aber selbst lobbyspezifische Ressourcen (Informationen, Netzwerke usw.). Eine erfolgreiche Lobbytätigkeit ruht auf vier Säulen: Monitoring, Netzwerkarbeit, Politikberatung, Öffentlichkeitsarbeit.

\section{Die erste Säule: Monitoring}

Monitoring meint die kontinuierliche Beobachtung von Politikprozessen. Dabei gilt es Politik sowohl hinsichtlich ihrer Inhalte ("policy «) als auch hinsichtlich der Entscheidungsstrukturen und Abläufe (»politics «) im Blick zu haben. Es geht einerseits darum, frühzeitig auf Themenkonjunkturen, Programmüberlegungen, Reformvorhaben usw. aufmerksam zu werden und sich so einen Informationsvorsprung zu sichern. Nur wer frühzeitig wahrnimmt, was in Rat und Verwaltung, in Gremien, Arbeitsgemeinschaften, Koordinierungskreisen oder der Öffentlichkeit gedacht und diskutiert wird, hat (a) überhaupt ausreichend Zeit um zu reagieren und kann (b) zu einem möglichst frühen und damit erfolgversprechenderen Zeitpunkt intervenieren. Gleichzeitig gilt es jederzeit über Entscheidungssystem und Entscheidungsprozesse informiert zu sein. Wer ist an welchen Stellen mit welchen Themen befasst? Wer hat welchen Einfluss auf die Behandlung des Themas und die zu treffenden Entscheidungen? Wie sind die Entscheidungswege? Wer sind potentielle Verbündete oder Gegner in einem Themenfeld?

\section{Die zweite Säule Netzwerkarbeit}

Netzwerkarbeit bezeichnet den für ein erfolgreiches Lobbying unverzichtbaren Aufbau von Beziehungen. Politische Netzwerke zu schaffen, die einen raschen direkten Zugang zu Entscheidungsträgern eröffnen oder schnell gemeinsame Aktivitäten und Stellungnahmen organisieren lassen und die auf wechselseitigem Vertrauen beruhen, gehört zusammen mit Monitoring zu den notwendigen Vorfeldarbeiten erfolgreichen Lobbyings. Die Pflege von Kontakten zu Bürokratie und Mandatsträgern ist eine ständige Aufgabe. Es gilt durch persönliche Gespräche, Briefe, Mails und Telefonate Kontakt zu halten und über die Organisation von Empfängen, Tagen der offenen Tür, Fachtagungen usw. Foren des Austauschs und Kennenlernens zu schaffen. Nur wenn zwischen den Akteuren ein Verhältnis wechselseitigen Respekts und Vertrauens herrscht, ist gewährleistet, dass (a) Informationen frühzeitig an die jeweilige Interessengruppe fließen und (b) ihre Stellungnahmen entsprechend zur Kenntnis genommen werden. Dabei muss kommunale Netzwerkarbeit sowohl auf Mandatsträger wie Sozialverwaltung, als auch auf andere Anbieter sozialer Dienstleistungen sowie auf zivilgesellschaftliche Einbindung zielen. Denn im Rahmen von Governance-Strukturen gilt es fachlich zu trennen, zu kommunizieren und zu vereinbaren, wo und in welchen Umfang man Konkurrent ist und wo man Interessen besser gemeinsam verfolgt.

\section{Die dritte Säule: Politikberatung}

Politikberatung zielt auf Aufklärung und Information der politischen Entscheidungsträger durch fachliche, wissenschaftlich aufbereitete Stellungnahmen, Gutachten, Planungen und Konzepte. Wesentliche Grundlagen einer gelingenden Lobbyarbeit sind fachlich ausgereifte Stellungnahmen und Konzepte, die hohe Kompetenz in sozialen Fragen hinsichtlich Problemanalyse wie Problembewältigung deutlich machen. Auf Seiten der sozialarbeitspolitischen Akteure bedarf es eines ausgewiesenen Knowhows hinsichtlich sozialer Problemlagen, sozialplanerischer Instrumente sowie ebenso innovativer wie realisierbarer Ideen. Dabei kommt es stets darauf an, Stellungnahmen wie Gutachten sorgfältig zu erarbeiten (Vertrauenswürdigkeit), mit Zahlen und empirischem Datenmaterial zu unterfüttern und ebenso knapp wie verständlich zu formulieren (Kommunikationsfähigkeit).

\section{Die vierte Säule: Öffentlichkeitsarbeit}

Öffentlichkeitsarbeit unterstützt und ergänzt Lobbyarbeit. Dabei geht es weniger um die Erzeugung von Druck bei entsprechender Mobilisierung von Öffentlichkeit. Den Druck auf die Politik über Medien und Öffentlichkeit zu erhöhen, wird in der Regel nur dann versucht, wenn Möglichkeiten des direkten Lobbying (persönliches Gespräch, Stellungnahme, Gutachten, Anhörung usw.) erschöpft erscheinen. Wichtiger ist eine kontinuierliche, begleitende Öffentlichkeitsarbeit (»Public Affairs Management «) zur Schaffung eines für die jeweiligen Klientengruppen, sozialarbeiterischen Interventionen und Projekte förderlichen Meinungsklimas. Es gilt das Ansehen der eigenen Organisation zu pflegen und die Aufklärung der Öffentlichkeit hinsichtlich Problemwahrnehmung und Wertorientierung voranzutreiben. Dies gelingt weniger durch ausgefeilte Web-Auftritte und Hochglanzjahresberichte, sondern eher durch persönliche Kontaktpflege, Präsenz im Sozialraum und zivilgesellschaftliche Einbindung.

\section{Warnung}

Kommunales Lobbying ist aber nur dann professionell zu nennen, wenn die Strategien und Instrumente der politisch Handelnden an normativen Leitideen wie Empowerment, Partizipation und Sozialer Gerechtigkeit orientiert sind. Ohne eine kritische ethisch-politische Reflexion und Einordnung verkümmert kommunales Lobbying schnell zu einer Klientelpolitik des Verbands- und Einrichtungsegoismus. Politisches Handeln in der Sozialen Arbeit kann und darf sich der Spannung zwischen organisationsbezogenen Eigeninteressen, fachlichen Idealen und advokatorisch zu vertretenden Klienteninteressen nicht entziehen. Denn gerade in der Doppelfunktion von Dienstleistungsorganisation und Sozialanwalt liegt ein Alleinstellungsmerkmal der freien Wohlfahrtspflege. Der fachliche Diskurs über das Politikmachen der Sozialen Arbeit sollte dringend intensiviert werden.

\section{Literatur:}

Benz, A. u. a. (Hg.) 2007: Handbuch Governance. Theoretische Grundlagen und empirische Anwendungsfelder, Wiesbaden.

Bogumil, J./Holtkamp, L. 2006: Kommunalpolitik und Kommunalverwaltung, Wiesbaden. Dahme, H.-J. u. a. 2008: Lehrbuch Kommunale Sozialverwaltung und Soziale Dienste. Grundlagen, aktuelle Praxis und Entwicklungsperspektiven, Weinheim, München.

Dettling, W. 2001: Die Stadt und ihre Bürger. Neue Wege in der kommunalen Sozialpolitik. Grundlagen - Perspektiven - Beispiele, Gütersloh.

Falk, S. u. a. (Hg.) 2006: Handbuch Politikberatung, Wiesbaden.

Kleinfeld, R. u. a. (Hg.) 2007: Lobbying. Strukturen, Akteure, Strategien, Wiesbaden. Lallinger, M./Rieger, G. (Hg.) 2007: Repolitisierung Sozialer Arbeit. Engagiert und professionell, Stuttgart (Hohenheim).

Popple, P. R./Leighninger, L. 1998: Policy-Based Profession. An Introduction to Social Welfare Policy for Social Workers, Needham Heights, MA. 